\title{
Simultaneous Latent Fingerprint Detection
}

\author{
Rozita Mohd Yusof ${ }^{1}$, Norrozila Sulaiman ${ }^{2}$ \\ ${ }^{1,2}$ (Faculty of Computer System and Software EngineeringUniversiti Malaysia Pahang, Malaysia)
}

\begin{abstract}
In this paper we present a novel approach for matching simultaneous latent fingerprint to plain fingerprint. In the experiment, we tested 160 simultaneous latent print images that are equal to 10 classes from 240 simultaneous impressions in the IIITD SLF Database and match them with a gallery of optical slap fingerprints. The Gallery of Optical is used as references to the accuracy of matching latent fingerprint to optical fingerprint. The experiment results demonstrate that the proposed method with some improvement to the matching score, FRR percentage, sensitivity and the specificity.
\end{abstract}

Keywords - Matching, simultaneous latent fingerprint, matching score.

\section{INTRODUCTION}

One of the oldest and most important evidence categories in forensic science and identification is fingerprint. Since the late nineteenth century, Federal Bureau of Investigation (FBI) fingerprint examiners determined that the latent fingerprint was of value for identification purposes (Robichaud 2006; Tico \& Kuosmanen 2000). Police around the world used fingerprint identification method to identify suspected criminals as well as the victims of crime. The pattern of the friction ridges on the surface of a fingertip is unique to each individual and does not change over a human lifetime (Wikipedia 2012).

In Northern Canada, over two million sets of fingerprints in the oldest fingerprint repository held by Canada's Royal Canadian Mounted Police (R.C.M.P.) which established in 1911. An Automated Fingerprint Identification System (AFIS) called Videofile which is possible to store large libraries of Canadian fingerprint image file on magnetic tape is introduced in 1970 in Canada (Chouinard 2011, p.18). In 1915, the first professional organization of forensic was established, called the International Association for Identification. This organization was launched the first professional certification program for a forensic scientist, the IAI's Certified Latent Print Examiner program in 1977. This program was issuing certification to those meeting rigorous criteria and canceling certification for serious error (Morgan 2007).

A latent fingerprint is lifted from the impression of the friction ridges deposited on the surface of an object or a wall (Wikipedia 2012; Jain \& Feng 2011). Typically, latent fingerprints are in bad condition or quality and poor in information content rather than rolled and plain impressions are obtained either by scanning the inked impression on paper or by using live scan devices (Jain \& Feng 2011). The big mistake of a latent examines can arise problems such as false reject rate (FRR) and false accept rate (FAR). When the subject's rolled or plain fingerprints are truly in the database but the latent examiner fails to identify the correct matched, a false accept will occur. The result of false accepts can cause the wrongful conviction of innocent persons. Criminals may not be apprehended if false rejects of the actual fingerprint happen. These are generally deemed as serious mistakes.

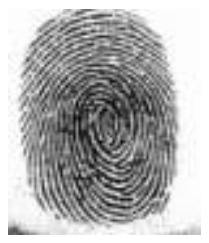

(a)

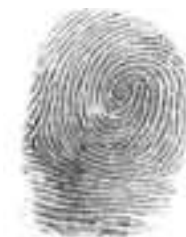

(b)

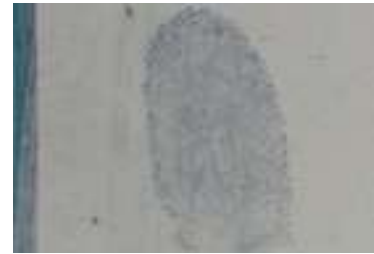

(c)

Figure 1.1: The example of fingerprint image obtained using different method. (a) Rolled impression image,

(b) live-scan, and (c) Typical latent image (Dvornychenko \& Garris 2006).

Refer to the latent fingerprints condition and quality, the manual invention is needed to fix the clarity of ridge information and extract the minutiae. However, this is does not restrain the Automated Fingerprint Identification System (AFIS) is widely deployed in law enforcement agencies. The low quality image of latent fingerprint becomes the main problem in the minutiae extraction using AFIS. Image enhancement is an important process in order to fix the quality of the latent fingerprint image.

This paper is organized as follows. Section 2 describes the existing of preprocessing methods. Section 3 introduces the proposed method in details. Section 4 represents our experimental results. 


\section{RELATED WORKS}

\subsection{Preprocessing- Fingerprint Enhancement}

The existing techniques of fingerprint enhancement mostly use a contextual filter which is depending on the orientation and local ridge frequency (Aguilar et al., 2008). The context information consists of ridge continuity and regularity of the fingerprint image corrupted can be recovered using contextual information from the surrounding neighborhood. In our work, we demonstrate the utility of the Gabor Filter and Fast Fourier Transform to carry out the image enhancement.

A set band pass filters that remove the undesired noise and preserve the true ridge or valley structure is introduced by Hong et al. (1998) and Khalil et al. (2009) namely Gabor Filter. Gabor Filter detects the spurious minutiae is based on the region between them is brighter than the average brightness of foreground region. Shen et al. (2001) proposed a method based on Gabor features; each block is filtered using a Gabor filter with different directions. Yun and Cho (2006) proposed an adaptively preprocessing method to improve the quality image base on Hong et al. (1998) proposed which modeled the ridge and valley pattern as sine wave. High quality image has a strong ridge direction.

Teddy (2002) proposed a latent fingerprint enhancement method using spectral (frequency) analysis technique such as brightness, contrast and color map adjustment to examine the minutiae information. However, besides the spectral analysis, latent fingerprints need to use spatial image enhancement filtering to enhance the degraded fingerprint information from a variety of background patterns. From the approaches, this research proposed to use a combination of the Gabor Filter and Fourier transform to carry out the image enhancement which is proposed by Aguilar et al. (2008).

\subsection{Segmentation}

An initial study on latent simultaneous fingerprint impressions is also performed by Black (2006) to determine the error rate when points of similarity from non contiguous simultaneous impressions are treated in aggregate for comparison and identification purposes. Vatsa (2009) introduces the research problem of identifying simultaneous latent fingerprint impressions via cluster and presents a semi-automatic approach to process the impressions of any individual.

\subsection{Minutiae Extraction}

Minutiae extraction is known as postprocessing in the fingerprint recognition system. This processing is employed in order to detect the true minutiae and eliminate the spurious minutiae after the latent segmentation or enhancement phase. Kim et al. (2001) and Mahadik et al. (2009) suggested a post-processing algorithm based on orientation and flow of ridges as the key factor to avoid eliminating true minutiae. They used a count of pixel value transition at a point of interest in a $3 \times 3$ mask to detect minutiae. If the count equal to two, the point is a ridge ending. If the count is corresponding to six, then the point is ridge bifurcation. The majority of the proposed approaches (Xiao and Rafaat, 1995; Zhao and Tang, 2007; and Akram et al. 2008; Rozita and Norrozila, 2012) for image postprocessing are based on the properties of the Crossing Number (CN) used to eliminate spurious minutiae.

\subsection{Matching}

In general, AFIS is used for mate of latent print feasible by searching print matching through large fingerprint databases. AFIS cannot yet offer satisfactory accuracy for most of latent prints. Latent feature extraction with manually mark the features are not satisfactory in term of accuracy and speed. Paulino et. Al., 2010 improve latent to full fingerprint matching accuracy by combining manually marked (ground truth) minutiae with automatically extracted minutiae.

\section{PROPOSED METHOD}

In this paper, figure 1 presents an overview of the proposed method. This method consists of six stages which are explained briefly as follows:

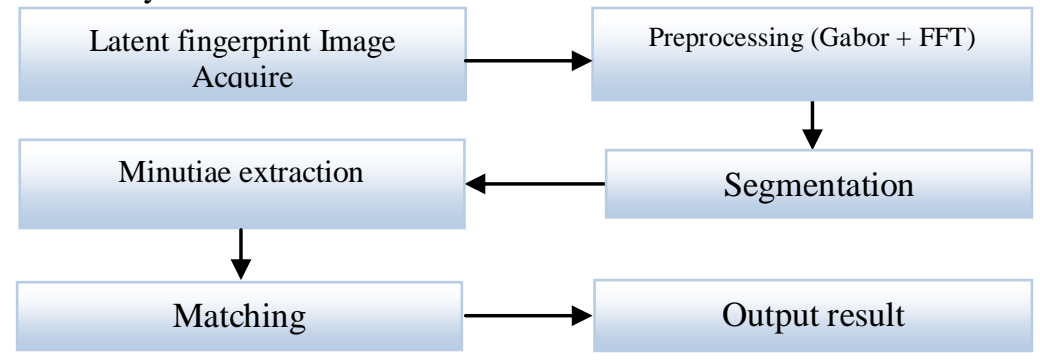

Figure 3.1: Overview of Proposed Method 


\subsection{Preprocessing}

Gabor filter is a set band pass filter or spatial domain filtering that remove the undesired noise and preserve the true ridge or valley structure. Gabor filters have significant signal properties for instance optimal joint space frequency resolution (Qian and Chen, 1996). Equation 1 represents the general form of the even symmetric Gabor as below:

$$
G(r, s)=\exp \left\{-\frac{1}{2}\left[\begin{array}{c}
r^{2}{ }^{2} \\
\delta_{r}{ }^{2}
\end{array}+\frac{s^{2}}{\delta_{s}{ }^{2}}\right]\right\} \cos (2 \pi f r)
$$

Where, f represents the ridge frequency and $\delta \mathrm{r} 2$ and $\delta \mathrm{s} 2$ determines the shape of the filter envelope.

Another approach that applied for performing enhancement completely in this study is Fourier domain which is based on 'root filtering' technique. In this approach, the image is divided into overlapping block and in each block. The enhanced image is obtained by

$$
\begin{aligned}
& I_{\xi n}(r, s)=F F T^{-1}\left\{F(t, u)|F(t, u)|^{k}\right\} \\
& F(t, u)=F F T(I(r, s))
\end{aligned}
$$

\subsection{Segmentation}

In this section, multi probe fusion framework for matching simultaneous latent impressions is proposed. Fig 2 shows the matching of simultaneous latent impression with optical gallery. The multi probe fusion at score level is performed. The Algorithm is explained as follows:

1) A simultaneous impression $S f i$ consists of multiple finger $f i, i$ is equal to 2, 3, and 4 or 5 . Each finger's $f i$ ( $i \epsilon$ $n$ ) region of interest (ROI) is manually marked and segmented.

2) A reference impression; optical-scan or plain latent print $S f r$ also consists of multiple impression of fingerprint $f r=2,3,4$ or 5 . The region of interest (ROI) for each $f r$ is also manually marked.

3) Before matching, $f i$ from $S f i$ and $f r$ from $S f r$ must be extracted in order to locate the minutiae and singular point using semi light out system. The examiner can add or marks the minutiae point manually after automate extract it using the system.

4) The matcher will combine and match the multiple fingerprint of the simultaneous impression $f i$ to $f r$ reference impression in order to create a probe that contains score matched.

5) The number of probe $\mathrm{P}($ fir $)$ is based on number of fi. Every probe has a set of scores matched with the optical-scan gallery using minutia-based matching and the distance of nearest neighborhood minutiae.

6) Each score matched in $\mathrm{P}($ fir $)$ is important in order to calculate the fusion score matched as shown in equation 1.

$$
f s=\frac{\sum p(f i r)}{n p}
$$

where $n p$ represents the number of probes.

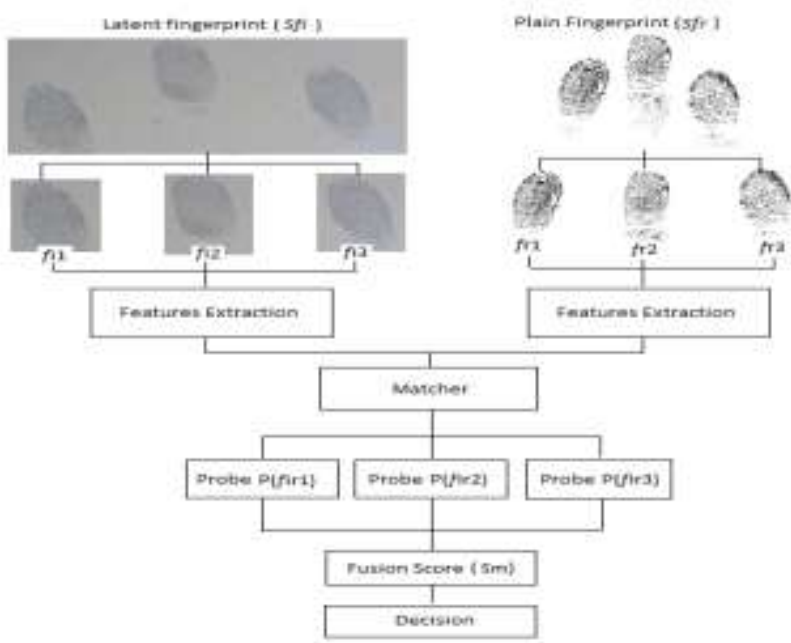

Figure 3.2: Demonstrating the stages involved in the proposed multiple probe approach. 


\subsection{Minutiae Extraction}

Crossing number $(\mathrm{CN})$ is employed as minutiae extraction technique in this study. By utilizing the skeleton image of the simultaneous latent fingerprint, $\mathrm{CN}$ has been implemented in order to identify the true minutiae using AFIS that shown in figure 3.3, table 3.1, table 3.2, and table 3.3.

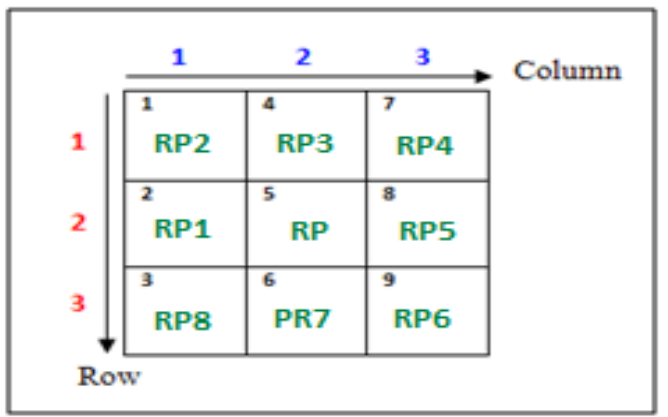

Figure 3.3: $3 \times 3$ windows of block pixels

To formulate the adjacency criterion for connectivity, we first introduce the notation of the neighborhood. For a pixel RP with the coordinates $(x, y)$ the set of pixels given by:

$$
N_{8}(R P)=N_{4} \cup\{(x+1, y+1),(x+1, y-1),(x-1, y+1),(x-1, y-1)\}
$$

From this we can infer the definition for 8-connectivity:

A pixel $R P$ is connected to a pixel $R P n$ if $R P$ is 8 -connected to $R P n$ or if $R P$ is 8 -connected to a third pixel which itself is connected to $R P n . n$ refer to $1,2, \ldots 8$ pixels. Figure 3.4 shows the crossing number $(\mathrm{CN})$ of ridge ending with $\mathrm{CN}=1$.

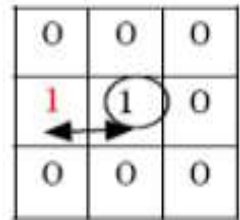

Figure 3.4: Ridge Ending

The result of the neighboring ridge segment is not necessarily appropriate to determine whether the minutiae are true or not. Therefore, the direction of the ridges block pixel is used to filter the true minutiae and eliminate the false and non-component of minutiae. Commonly, false ridge ending due to ridge breaks and scars [5]. The following are the rules to eliminate false end using the proposed algorithm:

1. The line rs is tracing first. If a break in a ridge is shorter than 15 pixels and no other ridge pass through it they will eliminate.

2. If they are very close with distance $|\mathrm{rs}| \leq 1 / 2 d$, and if $|\mathrm{rs}|>1 / 2 d$ with $\Delta \emptyset \mathrm{rs}>2 \mathrm{pi} / 3$, they will eliminate too.

3. For $1 / 2 \mathrm{~d}<|\mathrm{rs}| \leq d, \mathrm{r}$ and $\mathrm{s}$ are deleted if $\theta>2 \mathrm{pi} / 3$. For $d<|\mathrm{rs}| \leq 9 / 5 d$, they are deleted if $\theta>5 \pi / 6$.

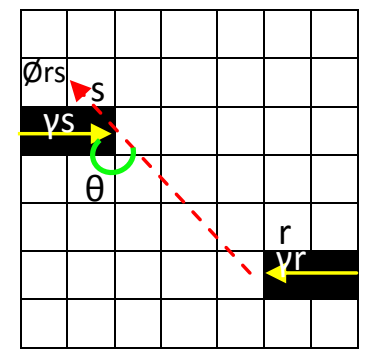

Figure 3.5: One of false ending pair structure

The point where the ridge splits into two or more branches is called as bifurcation. The Figure 3.6 shows the ridge bifurcation's crossing number is equal to 3 . 


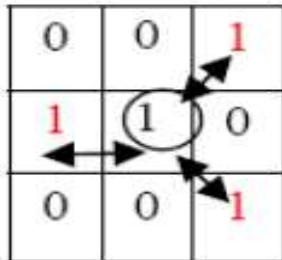

Figure 3.6: Shows one of ridge bifurcation structure with $\mathrm{CN}=3$

Not all the same $\mathrm{CN}=3$ is a true bifurcation. False bifurcation includes conventional bridges, crosses, and island. They need to be removed so that the true bifurcation can be detected. Bifurcation pairs are possibly false when:

1. Two bifurcation points are too close with distance $|\mathrm{rs}| \leq d$.

2. If $1 / 2 \mathrm{~d}<|\mathrm{rs}| \leq d$, the angle $(\theta)$ between $\gamma \mathrm{s}$ and Ørs need to be check. If $\theta<\mathrm{pi} / 8$ or $\theta<\mathrm{pi} / 12$, they must be eliminated.

3. The length of the branch is less than 20 pixels, and then the branch is removed.

Figure 3.7 shows a false ridge bifurcation structure with the illustration of the angle and distance of branches point.

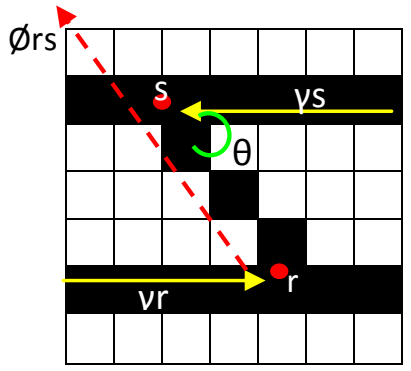

Figure 3.7: One of false ridge bifurcation structure

\subsection{Matching}

Matching between simultaneous latent fingerprints to plain fingerprint normally refers to similarity based on the type of minutia, orientation angle of each minutia, the distance of neighboring minutiae, and reference point. The similarity between two neighboring minutiae-based is computed as:

$$
S I_{m}=\frac{N M_{r}}{N M_{r}+N U_{r}} \cdot \frac{N M_{s}}{N M_{s}+N U_{s}}
$$

where, $\mathrm{NMr}$ is represents the number of neighboring minutiae of $\mathrm{r}$. $\mathrm{NM}_{\mathrm{s}}$ has denoted the number of neighboring minutiae of $s . \mathrm{NU}_{\mathrm{r}}$ is assigns as the number of unmatched neighboring minutiae of $\mathrm{r} . \mathrm{NU}_{\mathrm{s}}$ is referring to the number of unmatched neighboring minutiae of $s$.

Reference points consist of core, delta, and center point of reference are used in estimate the matched minutiae. Based on matched minutiae, the distance and angle of the reference points of the latent are computed and compared to 30 for distance and $\pi / 4$ for angle. The matching score is computed as:

$$
S M=S I_{m}+C V \cdot S_{r e f}
$$

where, $S M$ is referring to the matching score. $S I_{m}$ is assigned as the similarity between two neighboring minutiae-based. $C V$ is a constant value, empirically set at $0.03 . S_{\text {ref }}$ is indicated the number matching score based on matching reference points.

\subsection{Sensitivity and Specificity}

The performance has been measured based on the numbers of missing and spurious minutiae using clusters and non-clusters. Sensitivity is a metric measurement of the algorithm's ability to detect the genuine minutiae matched. A sensitivity of 1 or $100 \%$ means that the tests recognize minutiae as true minutiae. The higher the sensitivity, the less the true minutiae undetected. Equation 7 is the sensitivity, $S_{e n}$, formulation.

$$
S_{e n}=1-\frac{M_{m}}{M_{t}}
$$


where, $\mathrm{Mm}$ is missed minutiae and $\mathrm{Mt}$ is ground true minutiae. Equation 8 indicates the specificity, $S_{p e}$, is a metric measurement of the algorithm's ability to test correctly predicted false minutiae.

$$
S_{p e}=1-\frac{M_{f}}{M_{t}}
$$

Where, $M_{f}$ is false minutiae and $M_{t}$ is ground true minutiae.

\subsection{False Reject Rate (FRR)}

The big mistake of a latent examines can arise problems such as false reject and false accept. When the subject's rolled or plain fingerprints are truly in the database but the latent examiner fails to identify the correct matched, a false accept will occur. The result of false accepts can cause the wrongful conviction of innocent persons. In this study, a constant value of FAR is used, $0.01 \%$. The FRR is computed as equation 9.

$$
\mathrm{FRR}=\frac{M_{t y}}{T_{S L F}} \times 100 \%
$$

Where, $M_{t r}$ is the number of genuine latent fingerprints and $M_{t}$ is the total of latent fingerprints that is tested.

\subsection{IIITD-Simultaneous Fingerprint Database}

IIITD Simultaneous Latent Fingerprint database is prepared using the camera setup consists of a USB programmable UEye camera that has a resolution of 3840 x 2748. It has a 1/2" CMOS sensor and captures at a maximum rate of 3 frames per second (Image Analysis and Biometrics Lab, 2011). This camera setup can minimize the error introduced during this lifting procedure by captures the dusted fingerprint directly. In a semi controlled environment, multiple samples of various finger combinations from 15 subjects are collected with a ceramic tile as the background. Using Cross matches L-Scan Patrol, two sets of mated optical slap fingerprints $(4+4+2$ fingers) are captured for all 15 subjects. The number of simultaneous impressions is 240 prints and the number of optical slap impressions is 30 prints second (Sankaran, Vatsa \& Singh 2002).

\section{EXPERIMENTAL RESULTS}

In the experiment, we tested 160 simultaneous latent print images that are equal to 10 classes from 240 simultaneous impressions in the IIITD SLF Database and match them with a gallery of optical slap fingerprints. The effectiveness of the propose algorithm is evaluated. The optical images as the baseline accuracy of the database are provided in order to match the individual latent prints. The results of the fusion score are constructed upon it.

The region of interest (ROI) for the latent print is manually marked. The system automated marking the singular points and minutiae. The score matched was computed based on the matched minutiae for each pair of fingerprints being matched. We have shown that the accuracy match of simultaneous latent print can be improved by utilizing cluster's image. The experiment result exposed the decrease of False Reject Rate (FRR) using clusters is obtained $0.198 \%$ rather than non-clusters, $0.31 \%$ as shown in table 4.1 .

Table 4.1: The results of FRR, $S M, S_{p e}$, and $S_{e n}$ using clusters and non-cluster image approach.

\begin{tabular}{|c|c|c|c|c|}
\hline Probe & FRR (\%) & $\begin{array}{c}\text { SM } \\
(\text { Ave })\end{array}$ & $\begin{array}{c}\text { Spe } \\
(\text { Ave })\end{array}$ & $\begin{array}{c}\text { Sen } \\
(\text { Ave })\end{array}$ \\
\hline Cluster (Proposed) & 0.198 & 290.97 & 0.8702 & 0.9962 \\
\hline $\begin{array}{c}\text { Non-Cluster } \\
\text { (VeriFinger) }\end{array}$ & 0.315 & 154.01 & 0.699 & 0.9508 \\
\hline
\end{tabular}

The detail percent of FRR for each class is represented in fig. 4.2. Besides that, the score matched is increased using the clusters image as shown in Fig 4.1. The averages of Sen and Spe for clusters and non-clusters image are illustrated in Fig 4.3. 


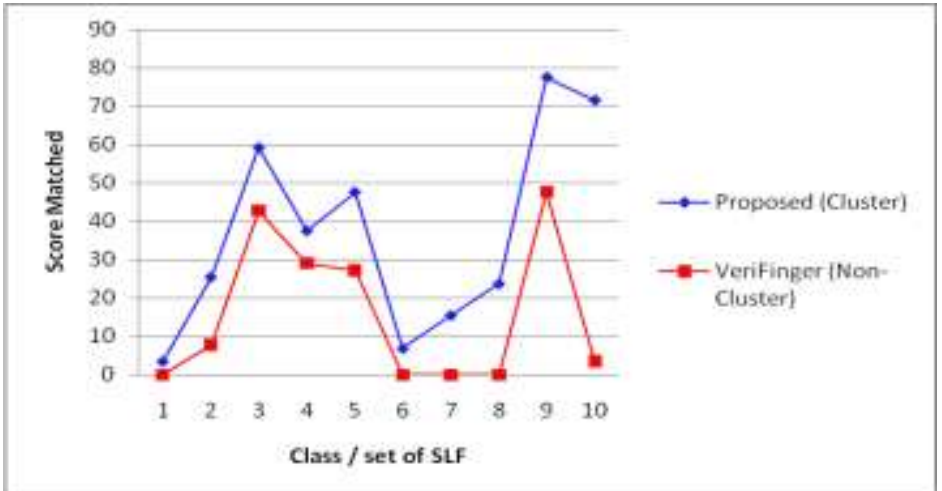

Fig. 4.1: Score match for clusters and non-clusters image

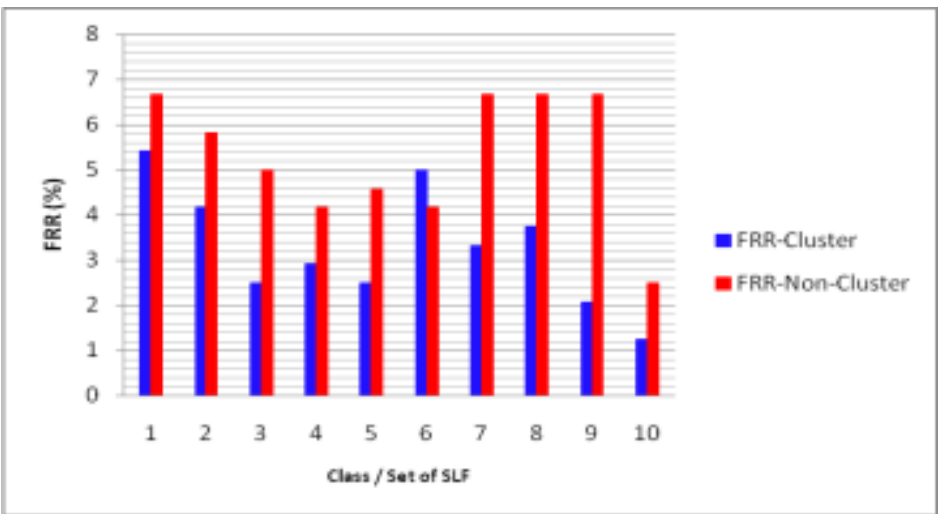

Fig. 4.2: FRR uses clusters and non-clusters image.

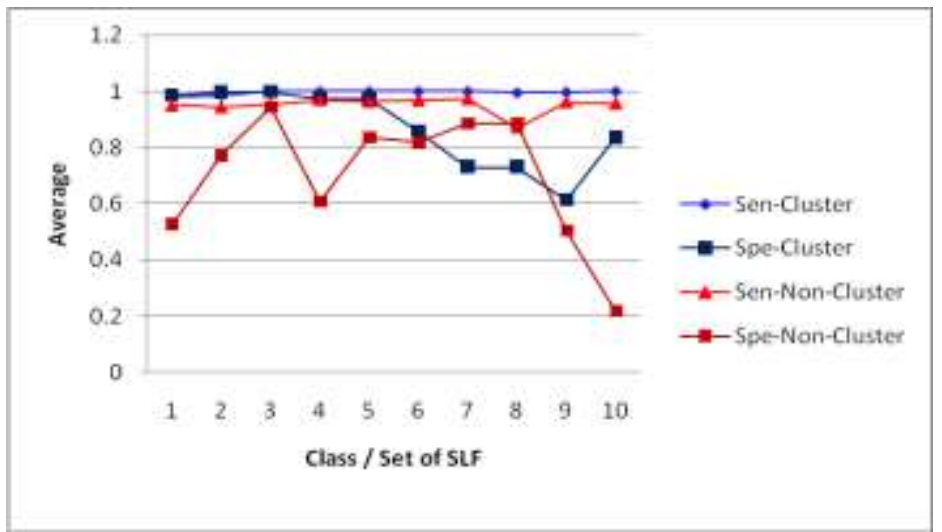

Fig. 4.3: Average result of Sen and Spe for clusters and non-clusters image

\section{CONCLUSION}

Poor quality is a big issue to the latent matching. A fully automatic system cannot offer the satisfaction of the performance due to their quality and manual intervention is still needed in order to improve the latent matching performance. We used manually marked ROI to extract the minutiae and singular point of the latent print. The FRR can be decreased and the score matched will be increased using the clusters image framework. The accuracy of the matching process is measured based on sensitivity, specificity, score matched, and FRR performance. The matching is high accuracy when the sensitivity is 1 , specificity and score matched are high and low of FRR.

\section{ACKNOWLEDGEMENTS}

This research work is supported by university grant, Grant Research Scheme (GRS), Universiti Malaysia Pahang, Malaysia. 


\section{REFERENCES}

[1] Sean Robichaud, 2006, The Use of Fingerprint Evidence in Criminal Cases, viewed 8 February 2012, http://knol.google.com/k/theuse-of-fingerprint-evidence-in-criminal-cases\#

[2] Wikipedia, the free encyclopedia, 2012. Fingerprint, viewed 9 February 2012, http://en.wikipedia.org/wiki/Fingerprint

[3] Eric Chouinard, 2011, Gazette - Big Impression, Celebrating 100 years of fingerprint identification in Canada, Gazette, Vol.73, No. 1, Page.18-19.

[4] Elizabeth Morgan. 2007, Fingerprinting in Forensic Science, viewed 9 February 2012, http://EzineArticles.com/410615

[5] Anil Jain and Jianjiang Feng, 2011, Latent Fingerprint Matching, IEEE transaction on Pattern Analysis and Machine Intelligence, Vol. 33, No.1, pp. 88-99.

[6] Hillary Lauren Moses, 2003, A Critical Comparison of Black Fingerprint Powder Formulations to Improve Latent Fingerprint Clarity and Contrast on Common Substrates, thesis, B.S Genetics, University of California, Davis.

[7] Rozita M.Y. and Norrozila S., 2012, Postprocessing Algorithm for Security Features Extraction, International Conference on eLearning and e-Technologies in Education (ICEEE), 2012, pp. 219-222. ISBN: 978-1-4673-1679-8.

[8] Marius Tico and Pauli Kuosmanen, 2000, An Algorithm for Fingerprint Image Processing, vol. 2, pp. 1735-1739, Pacific Grove, California, USA.

[9] David Heath. The Seattle Times June 07, 2004. Bungled fingerprints expose problems at FBI, viewed 9 February 2012, http://seattletimes.nwsource.com/html/localnews/2001949987_fingerprint07m.html

[10] S. Malathi and C.Meena, 2010, Partial fingerprint matching based on SIFT Features”, proceeding on (IJCSE) International Journal on Computer Science and Engineering Vol. 02, No. 04, pp.1411-1414.

[11] V. N. Dvornychenko and Michael D. Garris, 2006, Summary of NIST Latent Fingerprint Testing Workshop, NISTIR 7377.

[12] Image Analysis and Biometrics (IAB) Lab, 2011, IIIT-D latent fingerprint database, IIIT-D.

[13] Feng Zhao and Xiaoou Tang, 2002, Preprocessing for Skeleton-Based Fingerprint Minutiae Extraction, CISST'02 International Conference.

[14] D. Maltoni, D. Maio, A. K. Jain and S. Prabhakar, 2009. Handbook of fingerprint Recognition, 2nd ed, Springer-Verlag.

[15] S. Huckemann, T. Hotz, and Munk, 2008, Global Models for the Orientation Field of Fingerprints: An Approach Based on Quadratic Differentials", vol. 30, no. 9, pp. 1507-1519, IEEE Trans. PAMI.

[16] J. Zhou and J. Gu, 2004, Modeling orientation fields of fingerprints with rational complex functions; Pattern Recognition, vol. 37, pp. 389-391, 2004

[17] B. G Sherlock and D. M. Monro, 1993, A Model for Interpreting Fingerprint Topology”, Pattern Recognition, Vol. 26, no. 7, pp.1047-1055

[18] J. Feng and A. K. Jain, 2009, FM Model Based Fingerprint reconstruction from Minutiae Template, proceeding in ICB.

[19] Anush Sankaran, Mayank Vatsa and Richa Singh, 2012, On Simultaneous Latent Fingerprint Matching, IIITD-TR-2012-002.

[20] A. Paulino, Jain, A.K., J. Feng. 2010. Latent Fingerprint Matching: Fusion of Manually Marked and Derived Minutiae. Conference on Graphics, Patterns and Images (SIBGRAPI), 2010 23rd SIBGRAPI.

[21] Mohammed S. Khalil, Dzulkifli, M, and Qais, A.N. 2009. Fingerprint Verification using the texture of Fingerprint Image. The proceeding of Second International Conference on Machine Vision (ICMV). ISBN: 978-0-7695-3944-7/10.

[22] S. Mahadik, K. Narayan, D.V. Bhoir, and Darshana, S. Access Control System Using Fingerprint Recognition. Proceedings of International Conference on Advances in Computing, Communication and Control (ICAC3). 2009.ACM 978-1-60558-351-8.

[23] K. Teddy. 2002. Fingerprint Enhancement by Spectral Analysis Technique. The proceeding of 31st Applied Imagery Pattern.Recognition Workshop. Washington D.C.

[24] M. U. Akram, A. Tariq, and S. A. Khan. 2008. Fingerprint Image: Pre and Post Processing. The proceeding of International Journal of Biometrics (IJB). Vol. 1, no. 1.

[25] Q. Xiao and H. Rafaat. 1995. Fingerprint image Postprocessing: A Combined Statistical and Structural Approach. The proceedings of IEEE Transaction on Pattern Recognition. Vol. 28, no. 11, pp. 1657-1672.

[26] L. Hong, Y. Wang, A. K. Jain. 1998. Fingerprint image enhancement: Algorithm and performance evaluation. The proceeding of IEEE Transaction on Pattern Analysis and Machine Intelligence (PAMI). Vol. 21, No. 4 pp. 777-789.

[27] F. Zhao and X. Tang. 2007. Preprocessing and Postprocessing for Skeleton-based Fingerprint Minutiae Extraction. The proceeding of Pattern Recognition. Vol. 40, pp. 1270-1281.

[28] G. Aguilar, G. Sánchez, K. Toscano, M. N. Miyatake, and H. P. Meana.. Automatic Fingerprint Recognition System Using Fast Fourier Transform and Gabor Filters. The proceeding of Scientifica. 2008, Vol. 12, no. 1, pp. 9-16.

[29] M. Vatsa, R. Singh, A. Noore and K. Morris. Simultaneous Latent Fingerprint Recognition: A Preliminary Study. The proceedings of IEEE Transaction on Pattern Analysis and Machine Intelligence (PAMI). 2009. ISBN: 978-1-4244-5020-6.

[30] E.-K. Yun and S.B. Cho. Adaptive Fingerprint Image Enhancement with Fingerprint Image Quality Analysis. The proceedings of International conference of Image and Vision Computing. 2006. Vol. 24, pp. 101-110. 